The Journal of Animal \& Plant Sciences, 31(1): 2021, Page: 108-120 ISSN (print): 1018-7081; ISSN (online): 2309-8694

\title{
GENETIC ASSESSMENT OF CHLOROPHYLL $A$ AND $B$, CAROTENOIDS AND STOMATAL CONDUCTANCE IN LEAF TISSUE OF UPLAND COTTON IN WATER STRESS CONDITIONS
}

\author{
A. Rehman ${ }^{1}$ and M. T. Azhar ${ }^{1,2 *}$ \\ ${ }^{1}$ School of Agricultural Sciences, Zhengzhou University, Zhengzhou, Henan 450001, China \\ ${ }^{2}$ Department of Plant Breeding and Genetics, University of Agriculture, Faisalabad (Pakistan) \\ *Corresponding author's email address: tehseenazhar@gmail.com
}

\begin{abstract}
Water stress is a major threat to crop production in climate change scenario. Leaf physiological traits play an important role in maintenance of plant physiological processes under water stress. Therefore, the present study was conducted to characterize the leaf physiological traits of cotton under water stress as well as investigate the genetics of their inheritance. Sixty cotton hybrids along with parents were sown in field conditions in triplicate following split plot design under normal and water stress conditions. Data were collected for leaf physiological traits i.e. Chlorophyll $a$ and $b$, carotenoids, stomatal conductance and transpiration rate. Crosses namely, CIM-482 × NIAB Karishma, MNH-93 $\times$ S-12, CRIS-134 × ACALA-1517-C, CP-15/2 × FH-1000 and CRIS-134 × CIM-506 exhibited higher estimates of heritability, heterosis and heterobeltiosis suggested the presence of potential for genetic improvement through breeding and selection. Analysis of variance revealed the presence of significant differences among the genotypes for all the studied traits. Beside the lines i.e. FH-900, MNH-93 and NIAB-111 and testers CIM-506, NIAB Karishma and MNH-129 were found to be good general combiner under water stress conditions. The preponderance of non-additive gene action for the traits mentioned in this manuscript depict that superior combinations in current study can be used in drought tolerant cotton varieties with early generation selection.
\end{abstract}

Key words: Chlorophyll contents; Cotton; Genetic analysis; Stomatal conductance

https://doi.org/10.36899/JAPS.2021.1.0199

Published online August 26, 2020

\section{INTRODUCTION}

Cotton is the most important fiber crop around the globe to fulfill the demand of textile industry based on natural fiber. Upland cotton accounts for more than $90 \%$ of the world's cotton production (Cai et al., 2017) and $2^{\text {nd }}$ important oil seed crop in the world after soybean (Mahawar et al., 2017). In climate change scenario, water stress is the major threat to the world food security. Extent of water stress depends upon several factors i.e. moisture storing capacity of soils, evaporative demands and distribution of rainfall (Brauman et al., 2013). Continues appraisals are performed from two decades to understand genetic mechanisms involved in water stress tolerance in crop plants (Osakabe et al., 2014). For the development of water stress tolerance, understanding of physiological mechanisms and genetic control is most important. Likewise, water stress results in reduction of photosynthetic activity due to decrease in leaf expansion and impairs photosynthetic apparatus (Kalaji et al., 2016). Chlorophyll is the main light absorbing pigment. In higher plants, there are two types, chlorophyll a, the main pigment, and chlorophyll $b$ that together with carotenoids compounds the accessory pigments. Chlorophylls are packed in protein complexes forming what is called the photosynthetic complexes. These are constituted by an antenna complex which absorbs the majority of light energy and the photosystems, where the light is transformed by a photochemical reaction in chemical energy (Björn et al., 2009). A considerable reduction in contents of chlorophyll, net photosynthesis and carotenoids was observed in wheat (Abid et al., 2018). Carotenes are very responsive to oxidative damage. In green plants, B-carotene plays a vital protective role by quenching triplet chlorophyll, hence prevents the production of singlet oxygen and secure the plant from oxidative damages (Chourasia, 2017). They are separated into the hydrocarbon carotenes, such as lycopene and $\beta$-carotene or xanthophylls, typified by lutein (Jaleel et al., 2007). Oxidative damage caused by drought stress in the plant tissue is eased by an intensive action of both enzymatic and non-enzymatic antioxidant systems. These include $\beta$-carotenes, ascorbate, $\alpha$ tocopherol, reduced glutathione and enzymes including superoxide dismutase, peroxidase, ascorbate peroxidase, catalase, polyphenol oxidase and glutathione reductase (Prochazkova et al., 2001). Water stress leads to reduced stomatal conductance which ultimately results in reduced rate of photosynthesis (Miner et al., 2017). Likewise, damage to oxidative system of plant tissues is increased due to water stress ( $\mathrm{Li}$ et al., 2017). Closure of stomata 
under water stress conditions lead to reduced photosynthesis, and significant reduction in photosynthetic rate, $\mathrm{CO}_{2}$ uptake and transpiration rate (Karimi et al., 2015) and genotypes with stable performance of these traits may tolerate harsh conditions of water stress with minimal economic loss (Luo et al., 2016; Ullah and Zafar 2006; Fang and Xiong, 2015). Maqsood et al. (2018) reported additive gene action for stomatal conductance where as Ozdemir and Sade, (2019) observed non-additive gene action for stomatal conductance, transpiration rate and chlorophyll contents. Parveen et al. (2019) reported similar findings for chlorophyll and carotenoids contents. The combining ability and heterosis are important genetic parameters for determining breeding values of parents (Sarwarkar et al., 2015).

The most sustainable way to produce cotton under water stress conditions in climate change scenario is the development of water stress tolerant genotypes. As yield is dependent on photosynthetic efficiency of leaves, therefore understanding of inheritance pattern of leaf physiology is crucial to develop water stress tolerant genotypes. There are two pre-requisites for the development of water stress tolerant germplasm. Firstly, the presence of genetic variability for certain traits of interest, and secondly the identified traits must be genetically controlled. Under these circumstances, major objective of the study was to investigate the genetic variability and inheritance pattern of leaf physiological traits under normal and water stress conditions.

\section{MATERIALS AND METHODS}

Experimental site and location: The current study was carried out in the experimental area of University of Agriculture, (latitude $31^{\circ} 25^{\prime} \mathrm{N}$, longitude $73^{\circ} 09^{\prime} \mathrm{E}$ and altitude $184.4 \mathrm{~m}$ from sea level) Faisalabad, Pakistan whilst average temperature $35{ }^{\circ} \mathrm{C}$ with $40 \%$ humidity during crop season.

Plant material: Germplasm used herein was collected from Cotton Research Station (CRS), Multan; Central Cotton Research Institute (CCRI), Multan; Nuclear Institute for Agriculture and Biology (NIAB), Faisalabad; Cotton Research Institute (CRI), Faisalabad and Cotton Research Station (CRS), Bahawalpur-Pakistan. Fifty genotypes were screened in glass house with 80, 60, 40 an $20 \%$ of field capacity (Rehman et al., 2017). After screening, ten water stress tolerant cotton lines namely CP-15/2, NIAB-111, CIM-1100, BH-160, CRIS-134, FH-900, CIM-446, CIM-482, MNH-93, and CIM-707 and six water stress susceptible testers i.e. NIAB Karishma, CIM-506, FH-1000, MNH-129, Acala-1517C and S-12 were field planted during growing season of cotton 2014-15. Agronomic and other measures were taken to obtain healthy and better plant growth. Selected
16 cultivars then hybridized according to Line $\times$ Tester mating design at the time of flowering. At maturity, hybrid seeds from all the crosses stored till next crop season for plantation.

Experimental design and treatment: Sixty hybrids along with sixteen parents were planted in next cotton season 2015-16 with three replications following split plot design in two regimes i.e. control and water stress. From each genotype ten plants were planted in a row by maintaining row to row $75 \mathrm{~cm}$ and plant to plant distance of $30 \mathrm{~cm}$. Water stressed plants irrigated four times while eight irrigations designated as control (Kirda et al., 2005). In water stress, $1^{\text {st }}$ irrigation on germination stage, $2^{\text {nd }}$ on seedling establishment, $3^{\text {rd }}$ before squaring, while $4^{\text {th }}$ on peak flowering stage while normal plants were treated with 8 irrigations. All recommended production practices and plant protection measures were adopted during the experiment. Data were recorded on seven plants from each family on biochemical and physiological parameters in field as well as in laboratory conditions at maturity stage. The detailed protocol of each parameter is mentioned below,

Gas exchange parameters: Stomatal conductance and photosynthesis rate per plant were recorded three times from leaves of seven randomly selected plants from control and water stress conditions with the help of portable infrared gas analyzer (340 Bioscientific Ltd., UK). The data were recorded on fully expanded youngest leaves for gas exchange characteristics from 10:00 AM 12:00 PM.

Determination of chlorophyll contents and carotenes: $20 \mathrm{mg}$ fresh sample of young leaf tissue from each genotype was dipped in falcon tubes then pour $4 \mathrm{ml}$ of $100 \%$ methanol and incubated at $4{ }^{\circ} \mathrm{C}$ for one hour after wrapping the racks with aluminum foil to avoid light. At $4^{\circ} \mathrm{C}$, centrifuged the samples at $14,000 \mathrm{rpm}$ for $10 \mathrm{~min}$. Almost $3 \mathrm{ml}$ of sample was taken in cuvette and absorbance was measured at $470 \mathrm{~nm}$ (max. absorbance of carotenoids), $653 \mathrm{~nm}$ (max. absorbance of Chlorophyll $b$ ) and $666 \mathrm{~nm}$ (max. absorbance of Chlorophyll $a$ ) on a double beam spectrophotometer (Shimdtzu-190). According to Costache et al. (2012) the concentrations of chlorophyll $a$ and $b$ and carotenoids were calculated.

Chlorophyll $a=15.65$ A666 - 7.340 A653

Chlorophyll $b=27.05$ A653 - 11.21 A666

Carotene $=1000 \mathrm{~A} 470-2.860 \mathrm{Chl} a-129.2 \mathrm{Chl} b / 245$

Statistical analysis: Data were analyzed by different techniques followed by Steel and Dickey (1997) to verify the existence of genotypic differences for all the studied parameters. Same data were utilized to determine genetic components and combining ability by using Line $\times$ Tester analysis (Kempthorne, 1957) by using the statistical software Minitab 17. 


\section{RESULTS}

Genetic analysis of plant material: Analysis of variance following line $\times$ tester technique was analyzed to reveal the presence of genetic variations among plant material under normal and water stress field conditions. Biometrical analysis following line $\times$ tester technique to evaluate the genetic variation in cotton plants under normal and water stress conditions revealed that significant differences $(\mathrm{P} \leq 0.01)$ were observed among 76 genotypes for all the traits studied i.e. stomatal conductance, transpiration rate, chlorophyll $a$, chlorophyll $b$ and carotenoids (Table 1). Means square due to GCA effects (parents) and specific combining ability (crosses) were differed highly significant for all parameters in both conditions. Highly significant differences were observed for interaction due to parents $v s$ crosses whereas non-significant differences $(\mathrm{P} \geq 0.05)$ were noted for chlorophyll $b$ while highly significant for all of traits in water stress conditions. Significant differences were observed in lines and testers for physiological and leaf gas exchange traits except stomatal conductance in testers almost similar trend was found in water stress conditions except chlorophyll $a$ and transpiration rate. Highly significant differences were observed in interaction of lines and testers for the traits included in this study.

Estimation of genetic components and heritability: SCA variance $\left(\sigma^{2} \mathrm{sca}\right)$ in normal and water stress conditions is higher as compared to the GCA variance ( $\sigma^{2}$ gca) for all studied traits, indicating the presence of non-additive gene action in inheritance (Table 2). Positive sign gives us the information about the direction of dominance towards superior parent, whereas negative sign indicate that dominance was directed towards lower parent. The ratio of general to specific combining ability variance $\left(\sigma^{2} \mathrm{gca} / \sigma^{2} \mathrm{sca}\right)$ was recorded below unity, exhibited that studied traits are inherited by non-additive gene action in normal water conditions while similar results were found in water stress conditions. This table also showed that all parameters had higher narrow sense heritability values in both conditions. A high heritability index of $0.993,0.961,0.943$ indicated that selection breeding might be progressive for genetic improvement. Moreover, high narrow heritability indicating the possibility of progress from selection.

General combining ability: The numerical values assigned to ten lines and six testers under normal and water stress conditions indicated their general combining ability (GCA) for the traits (Table 3). In normal water conditions, estimates comparison showed that NIAB-111 showed positive value for stomatal conductance (0.10), chlorophyll $a$ (0.01) and carotenoids (0.01) exhibited good GCA for these characters while poor combiner for transpiration rate (-1.18) and chlorophyll $b(-0.04)$ while under waters stress conditions NIAB-111 displayed positive values and good GCA effects for stomatal conductance $(0.16)$, transpiration rate $(0.04)$ and carotenoids (0.02). Due to greater magnitude for stomatal conductance (0.45) and carotenoids (0.01), CP-15/2 displayed its superiority for GCA whilst poor combiner for transpiration rate $(-0.31)$, chlorophyll $a(-0.01)$ and chlorophyll $b(-0.02)$ whilst in water stress exhibited highest positive values for transpiration rate $(0.50)$ only. Line BH-160 was found superior general combiner for stomatal conductance (0.34), chlorophyll $a(0.001)$ and chlorophyll $b$ (0.02) while exhibited poor combiner for remaining traits whilst under water stress showed good GCA only for transpiration rate (0.79) and chlorophyll $b$ (0.03). CIM-1100 possessed good general combining ability for stomatal conductance $(0.18)$, transpiration rate (0.02) and carotenoids (0.03) in normal conditions whilst under water stress conditions only transpiration rate and carotenoids exhibited positive estimates while stomatal conductance was found to be -0.02 . CRIS-134 was identified as good combiner for transpiration rate (0.07) whilst under stress displayed opposite results. FH-900 showed good GCA for transpiration rate, chlorophyll $a$, chlorophyll $b$ and carotenoids with estimates of 1.14 , $0.001,0.07$ and 0.01 respectively while in water stress maximum positive GCA effects showed for chlorophyll $a$ (0.09), chlorophyll $b(0.06)$ and stomatal conductance (0.06). CIM-482 showed highest estimates of transpiration rate (0.28), chlorophyll $a(0.03)$, chlorophyll $b(0.01)$ and carotenoids $(0.001)$ and opposite results was found in water stress conditions. Among testers, CIM506 displayed positive GCA for stomatal conductance (0.001), chlorophyll $b(0.06)$ and carotenoids (0.01) while in stress conditions similar results were found. NIAB Karishma identified as good general combiner for transpiration rate and $\mathrm{CHL} a$ content under control conditions whereas transpiration rate, CHL $a$ contents and carotenoids also exhibited positives values under water stress conditions. Tester MNH-129 displayed negative GCA for all the traits except stomatal conductance (0.43) whilst in water stress conditions, higher values of GCA effects exhibited by all of traits except chlorophyll $a(-0.09)$ and carotenoids (-0.02). FH1000 showed good general combining abilities for transpiration rate (0.74), chlorophyll $b$ (0.001) and carotenoids (0.02) and almost similar results were showed in stress conditions i.e. attained highest positive coefficient for stomatal conductance (0.05), chlorophyll $a$ (0.04) and chlorophyll $b$ (0.001). Tester, Acala-1517-C exhibited good general combining ability for transpiration rate, chlorophyll $b$ and carotenoids with high GCA values i.e. $0.47,0.04$ and 0.03 respectively. While in water stress conditions, Acala-1517-C expressed its better GCA for chlorophyll $b(0.02)$ than other characters. 
Estimation of specific combining ability (SCA): Under normal water conditions, higher SCA effects for stomatal conductance was exhibited by BH-160 × CIM-506, MNH-93 $\times$ S-12, CIM-1100 $\times$ CIM-506, CP-15/2 $\times$ CIM-506 and CIM-1100 $\times$ MNH-129 (Table 4) whilst under water stress conditions combinations of CIM-482 $\times$ NIAB Karishma, CIM-1100 $\times$ S-12, CRIS-134 $\times$ MNH129 and CIM-707 $\times$ S-12 scored significant and highest coefficients of SCA, and appeared to be best specific combinations (Table 5). For transpiration rate, positive and significant SCA effects were found for FH-900 $\times$ CIM-506, CIM-707 $\times$ MNH-129, CIM-446 $\times$ ACALA1517-C, CIM-482 × S-12, CRIS-134 × NIAB Karishma and MNH-93 x MNH-129 under normal conditions whilst in water stress conditions, best specific combining ability was displayed by MNH-93 $\times$ S-12, NIAB-111 $\times$ NIAB Karishma, CP-15/2 × CIM-506 and CRIS-134 $\times$ FH-1000 due to having highest values. For Chlorophyll $a$, CIM-446 $\times$ NIAB Karishma, CIM-482 × CIM-506, FH-900 $\times$ NIAB Karishma, FH-900 $\times$ CIM-506, CIM446 x S-12 and CIM-707 x ACALA -1517-C expressed the best SCA estimates under normal water conditions, while the combinations of CRIS-134 $\times$ ACALA-1517-C, CIM-446 $\times$ S-12, FH-900 $\times$ FH-1000 and CIM-482 $\times$ CIM-506 presented significant and positive SCA effects under water stress. For Chlorophyll $b$, combinations of CP-15/2 × FH-1000, NIAB-111 $\times$ MNH-129, FH-900 $\times$ NIAB Karishma, MNH-93 $\times$ CIM-506 and NIAB $111 \times$ ACALA 1517-C exhibited significant positive SCA values under normal water conditions. Whilst under water stress conditions higher SCA effects was exhibited by BH-160 x MNH-129, CP-15/2 × FH-1000, NIAB-111 × ACALA-1517-C, NIAB-111 $\times$ MNH-129 and FH-900 $\times$ NIAB Karishma. For carotenoids, significant and positive SCA effects were found for MNH-93 $\times$ NIAB Karishma, CIM-482 $\times$ NIAB Karishma, CIM-707 $\times$ FH-1000 and $\mathrm{CP}-15 / 2 \times \mathrm{MNH}-129$ under water stress.
Estimation of heterosis and heterobeltiosis: Higher heterosis effects for stomatal conductance was showed by BH-160 × CIM-506, CP-15/2 x MNH-129 and CIM-1100 $\mathrm{x}$ MNH-129 under normal conditions and CIM-482 $\times$ NIAB Karishma under normal water conditions (Table 6 and Table 7). For transpiration rate, significant and positive heterosis were found for CRIS-134 $\times$ NIAB Karishma whilst under water stress conditions NIAB-111 $\times$ NIAB Karishma showed maximum heterotic estimates. For Chlorophyll $a$, combination of NIAB-111 $\times \mathrm{S}-12$ expressed the highest heterosis while CRIS-134 $\times$ ACALA-1517C exhibited highest estimates under normal and water stress conditions respectively. For Chlorophyll $b$, combinations of CIM-446 $\times$ ACALA-1517-C exhibited significant positive heterotic effects under both normal and water stress conditions. Whilst cross combination of CIM-446 $\times$ FH-1000 exhibited the highest estimates of heterosis while CRIS-134 × CIM506 presented highest estimates.

Under water stress conditions, for stomatal conductance, combination of CIM-482 × NIAB Karishma scored highest and significant coefficients of heterobeltiosis (Table 7). Combination MNH-93 $\times \mathrm{S}-12$ showed highest estimates for transpiration rate in normal conditions while CRIS-134 $\times$ NIAB Karishma in water stress conditions. NIAB-111 $\times \mathrm{S}-12$ showed positive estimates for chlorophyll $a$ while the combination of CRIS-134 $\times$ ACALA-1517-C presented highest, significant and positive heterobeltiosis effects in water stress conditions. Combination CIM-446 × ACALA1517-C exhibited highest estimates of heterobeltiotic for chlorophyll $b$ under both conditions. For carotenoids, maximum heterosis and heterobeltiosis estimates were exhibited by CRIS-134 × CIM-506.

Table 1. Mean squares of various quantitative traits of cotton grown under normal and water stress conditions.

\begin{tabular}{lcccccccccccc}
\hline \multicolumn{1}{c}{ Normal water conditions } & \multicolumn{4}{c}{ Water stress conditions } \\
\hline SOV & DF & SC & TR & CHL $a$ & CHL $b$ & CAR & SC & TR & CHL $a$ & CHL $b$ & CAR \\
Replications & 2 & 0.03 & 0.20 & 0.01 & 0.001 & 0.001 & 0.06 & 0.01 & 0.01 & 0.001 & 0.001 \\
Genotypes & 75 & $1.99^{* *}$ & $9.50^{* *}$ & $0.06^{* *}$ & $0.04^{* *}$ & $0.01^{* *}$ & $0.36^{* *}$ & $2.16^{* *}$ & $0.07^{* *}$ & $0.03^{* *}$ & $0.01^{* *}$ \\
Parents & 15 & $1.12^{* *}$ & $10.99^{* * *}$ & $0.05^{* *}$ & $0.03^{* *}$ & $0.02^{* *}$ & $0.36^{* *}$ & $0.66^{* *}$ & $0.07^{* *}$ & $0.02^{* *}$ & $0.01^{* *}$ \\
Crosses & 59 & $1.60^{* *}$ & $8.83^{* *}$ & $0.05^{* *}$ & $0.04^{* *}$ & $0.01^{* *}$ & $0.31^{* *}$ & $1.93^{* *}$ & $0.06^{* *}$ & $0.02^{* *}$ & $0.01^{* *}$ \\
Parents Vs & 1 & $38.32^{* *}$ & $27.13^{* *}$ & $0.71^{* *}$ & 0.001 & $0.04^{* *}$ & $3.27^{* *}$ & $38.45^{* *}$ & $0.58^{* *}$ & $0.34^{* *}$ & 0.00 \\
Crosses & 1 & & $0.35^{* *}$ & $4.37^{* *}$ & $0.06^{* *}$ & $0.03^{* *}$ & $0.03^{* *}$ & $0.32^{* *}$ & $0.35^{* *}$ & $0.08^{* *}$ & $0.01^{* *}$ & $0.02^{* *}$ \\
Lines & 9 & 0.15 & $0.44^{* *}$ & $0.01^{*}$ & $0.02^{* *}$ & $0.02^{* *}$ & $0.25^{* *}$ & 0.25 & 0.01 & $0.05^{* *}$ & $0.01^{* *}$ \\
Testers & 5 & $0.0 .0 .01^{* *}$ & $0.02^{* *}$ & $0.01^{* *}$ \\
Lines $\times$ Testers & 45 & $2.01^{* *}$ & $10.65^{* *}$ & $0.05^{* *}$ & $0.04^{* *}$ & $0.01^{* *}$ & $0.32^{* *}$ & $2.43^{* *}$ & $0.07^{* *}$ & 0.01 & 0.003 & 0.001 \\
Error & 150 & 0.08 & 0.07 & 0.01 & 0.003 & 0.008 & 0.05 & 0.11 & 0.01 & 0.01 \\
\hline
\end{tabular}

Where, DF stands for degree of freedom, SC- stomatal conductance, TR- transpiration rate, CHL $a$ - chlorophyll $a$, CHL $b$ - chlorophyll $b$ and CAR- carotenoids. 
Table 2. Genetic components of variation of various quantitative traits of cotton grown under normal and water stress conditions.

\begin{tabular}{|c|c|c|c|c|c|c|c|c|c|c|c|c|}
\hline \multirow[t]{2}{*}{$\begin{array}{l}\text { Plant } \\
\text { traits }\end{array}$} & \multicolumn{6}{|c|}{ Normal water conditions } & \multicolumn{6}{|c|}{ Water stress conditions } \\
\hline & $\sigma 2 \mathrm{gca}$ & $\sigma 2 \mathrm{sca}$ & $\sigma 2 A$ & $\sigma 2 D$ & $\begin{array}{l}\sigma 2 \mathrm{gca} / \\
\sigma 2 \mathrm{sca}\end{array}$ & Heritability & $\sigma 2 \mathrm{gca}$ & $\sigma 2 \mathrm{sca}$ & $\sigma 2 A$ & $\sigma 2 D$ & $\begin{array}{l}\sigma 2 \mathrm{gca} / \\
\sigma 2 \mathrm{sca}\end{array}$ & Heritability \\
\hline SC & -0.007 & -165.65 & -0.004 & -165.65 & 0.00004 & 0.908 & -0.0008 & -20.62 & -0.0004 & -20.62 & 0.00004 & 0.854 \\
\hline TR & -0.04 & -127.76 & -0.02 & -127.7 & 0.00031 & 0.899 & -0.009 & -112.6 & -0.004 & -112.6 & 0.00008 & 0.948 \\
\hline CHL a & -0.0002 & -43.15 & -0.0001 & -43.15 & 0.00001 & 0.943 & -0.0002 & -13.6 & -0.0001 & -13.6 & 0.00001 & 0.801 \\
\hline CHL $b$ & -0.0001 & -0.11 & -0.00006 & -0.11 & 0.00091 & 0.961 & -0.00001 & -34.73 & -0.00001 & -34.73 & 0.00001 & 0.875 \\
\hline CAR & 0.01 & -14.79 & 0.01 & -14.79 & 0.0006 & 0.993 & -0.00004 & -0.91 & -0.00002 & -0.91 & 0.00004 & 0.888 \\
\hline
\end{tabular}

Table 3. General combining abilities of various quantitative traits of cotton grown under normal and water stress conditions.

\begin{tabular}{|c|c|c|c|c|c|c|c|c|c|c|}
\hline \multirow[b]{2}{*}{ PARENTS } & \multicolumn{5}{|c|}{ Normal water conditions } & \multicolumn{5}{|c|}{ Water stress conditions } \\
\hline & $\mathrm{SC}$ & $\mathrm{TR}$ & CHL $a$ & CHL $b$ & CAR & $\mathrm{SC}$ & $\mathrm{TR}$ & CHL $a$ & CHL $b$ & CAR \\
\hline LINES & & & & & & & & & & \\
\hline NIAB-111 & 0.10 & -1.18 & 0.01 & -0.04 & 0.01 & 0.16 & 0.04 & -0.04 & -0.06 & 0.02 \\
\hline CP-15/2 & 0.45 & -0.31 & -0.01 & -0.02 & 0.01 & -0.21 & 0.50 & -0.04 & -0.04 & 0.001 \\
\hline BH-160 & 0.34 & -0.54 & 0.001 & 0.02 & -0.03 & -0.23 & 0.79 & -0.03 & 0.03 & -0.03 \\
\hline CIM-1100 & 0.18 & 0.02 & -0.05 & -0.03 & 0.03 & -0.02 & 0.21 & -0.06 & -0.01 & 0.001 \\
\hline CRIS-134 & -0.03 & 0.07 & -0.01 & -0.05 & -0.02 & 0.14 & -0.30 & 0.03 & -0.04 & 0.03 \\
\hline CIM-446 & -0.10 & -0.06 & -0.11 & 0.07 & -0.03 & -0.04 & 0.07 & -0.06 & 0.06 & 0.001 \\
\hline FH-900 & -0.09 & 1.14 & 0.001 & 0.07 & 0.01 & 0.06 & -0.28 & 0.09 & 0.06 & 0.001 \\
\hline MNH-93 & -0.35 & 0.16 & 0.10 & -0.02 & 0.02 & 0.14 & -0.09 & 0.08 & 0.001 & 0.001 \\
\hline CIM-707 & -0.24 & 0.41 & 0.03 & 0.01 & 0.01 & -0.17 & -0.35 & 0.001 & 0.001 & -0.01 \\
\hline CIM-482 & -0.25 & 0.28 & 0.03 & 0.01 & 0.001 & 0.18 & -0.58 & 0.03 & -0.01 & -0.01 \\
\hline $\begin{array}{l}\text { Standard Error } \\
\text { TESTERS }\end{array}$ & 28.38 & 26.62 & 14.69 & 0.81 & 8.6 & 10.18 & 23.81 & 8.25 & 13.18 & 2.14 \\
\hline CIM-506 & 0.001 & -1.5 & -0.04 & 0.06 & 0.01 & 0.12 & 0.09 & -0.06 & -0.01 & 0.06 \\
\hline NIAB Karishma & -0.11 & 0.45 & 0.1 & -0.04 & -0.01 & -0.05 & 0.07 & 0.06 & -0.02 & 0.03 \\
\hline MNH-129 & 0.43 & -0.46 & -0.05 & -0.06 & -0.02 & 0.05 & 0.41 & -0.09 & 0.001 & -0.02 \\
\hline FH-1000 & -0.23 & 0.74 & -0.03 & 0.001 & 0.02 & 0.05 & -0.27 & 0.04 & 0.001 & -0.01 \\
\hline S-12 & 0.19 & 0.35 & 0.07 & 0.01 & -0.03 & -0.02 & -0.20 & 0.06 & 0.02 & -0.05 \\
\hline ACALA-1517-C & -0.28 & 0.47 & -0.04 & 0.04 & 0.03 & -0.14 & -0.10 & 0.001 & 0.02 & 0.001 \\
\hline Standard Error & 40.78 & 36.27 & 20.77 & 1.14 & 12.16 & 14.39 & 33.67 & 11.67 & 18.63 & 3.03 \\
\hline
\end{tabular}


Table 4. Specific combining ability estimates of various quantitative traits of cotton grown under normal water conditions.

\begin{tabular}{|c|c|c|c|c|c|}
\hline Crosses & SC & TR & CHL $a$ & CHL $b$ & CAR \\
\hline NIAB-111 × CIM-506 & 0.26 & 1.00 & 0.03 & 0.06 & -0.05 \\
\hline CP- $15 / 2 \times$ CIM-506 & 0.98 & 0.93 & 0.08 & 0.03 & -0.01 \\
\hline BH-160 × CIM-506 & 1.55 & -1.84 & -0.15 & -0.06 & 0.07 \\
\hline CIM-1100 × CIM-506 & 1.04 & -1.73 & 0.06 & -0.06 & 0.05 \\
\hline CRIS-134 × CIM-506 & -0.91 & -0.82 & -0.18 & -0.02 & 0.01 \\
\hline CIM-446 × CIM-506 & -0.97 & -0.02 & -0.13 & -0.04 & -0.07 \\
\hline FH-900 × CIM-506 & -0.21 & 2.88 & 0.11 & 0.03 & 0.03 \\
\hline MNH-93 $\times$ CIM-506 & -0.62 & 0.22 & -0.02 & 0.13 & 0.07 \\
\hline CIM-707 × CIM-506 & -0.44 & -1.52 & 0.08 & 0.05 & 0.01 \\
\hline CIM-482 × CIM-506 & -0.69 & 0.90 & 0.14 & -0.12 & -0.11 \\
\hline NIAB-111 × NIAB Karishma & 0.30 & 1.49 & -0.19 & -0.09 & -0.01 \\
\hline CP-15/2 × NIAB Karishma & 0.27 & 1.35 & 0.01 & -0.13 & -0.03 \\
\hline BH-160 × NIAB Karishma & -1.19 & 0.88 & -0.13 & -0.09 & -0.06 \\
\hline CIM-1100 × NIAB Karishma & -0.87 & 0.92 & 0.09 & -0.03 & -0.04 \\
\hline CRIS-134 × NIAB Karishma & 0.18 & 1.77 & -0.02 & 0.01 & 0.03 \\
\hline CIM-446 × NIAB Karishma & 0.09 & -2.67 & 0.15 & 0.07 & -0.05 \\
\hline FH-900 × NIAB Karishma & 0.08 & -0.13 & 0.12 & 0.15 & 0.05 \\
\hline MNH-93 × NIAB Karishma & 0.01 & -3.88 & 0.06 & -0.05 & 0.09 \\
\hline CIM-707 $\times$ NIAB Karishma & 0.36 & 1.40 & 0.07 & 0.09 & -0.07 \\
\hline CIM-482 $\times$ NIAB Karishma & 0.77 & -1.14 & -0.17 & 0.08 & 0.09 \\
\hline NIAB-111 × MNH-129 & 0.06 & 0.94 & 0.06 & 0.17 & 0.05 \\
\hline $\mathrm{CP}-15 / 2 \times \mathrm{MNH}-129$ & 0.55 & -0.66 & 0.04 & -0.04 & 0.09 \\
\hline BH-160 × MNH-129 & 0.56 & -1.13 & 0.06 & 0.04 & 0.02 \\
\hline CIM-1100 × MNH-129 & 0.88 & -2.09 & 0.07 & 0.08 & -0.02 \\
\hline CRIS-134 × MNH-129 & 0.5 & -1.48 & 0.10 & -0.01 & -0.08 \\
\hline CIM-446 × MNH-129 & -0.36 & 1.02 & -0.01 & 0.01 & 0.01 \\
\hline FH-900 × MNH-129 & -0.97 & 0.29 & 0.04 & -0.13 & -0.02 \\
\hline MNH-93 × MNH-129 & -0.38 & 1.77 & -0.16 & 0.04 & -0.1 \\
\hline CIM-707 × MNH-129 & 0.04 & 2.38 & -0.23 & -0.12 & 0.04 \\
\hline CIM-482 × MNH-129 & -0.88 & -1.02 & 0.03 & 0.05 & 0.02 \\
\hline NIAB-111 × FH-1000 & -0.50 & -1.70 & 0.04 & -0.07 & 0.03 \\
\hline CP-15/2 × FH-1000 & -0.51 & 1.26 & 0.03 & 0.19 & -0.06 \\
\hline BH-160 × FH-1000 & -0.40 & 0.45 & 0.10 & 0.12 & 0.04 \\
\hline CIM-1100 × FH-1000 & -0.51 & 0.70 & -0.08 & 0.08 & -0.04 \\
\hline CRIS-134 × FH-1000 & 0.27 & -0.23 & 0.02 & -0.10 & -0.06 \\
\hline CIM-446 × FH-1000 & 0.71 & -0.54 & -0.06 & -0.03 & 0.01 \\
\hline FH-900 × FH-1000 & 0.53 & -1.07 & -0.21 & 0.06 & -0.03 \\
\hline MNH-93 $\times$ FH-1000 & 0.26 & 1.45 & 0.04 & 0.02 & 0.01 \\
\hline CIM-707 × FH-1000 & -0.12 & 0.32 & 0.04 & -0.09 & 0.09 \\
\hline CIM-482 × FH -1000 & 0.26 & -0.64 & 0.10 & -0.19 & 0.06 \\
\hline NIAB-111 × S-12 & -0.86 & -0.51 & 0.05 & -0.20 & 0.02 \\
\hline $\mathrm{CP}-15 / 2 \times \mathrm{S}-12$ & -1.34 & -1.45 & -0.15 & -0.11 & -0.05 \\
\hline BH-160 × S-12 & -1.03 & 0.82 & 0.04 & 0.07 & -0.09 \\
\hline CIM-1100 × S-12 & -0.44 & 0.99 & -0.04 & 0.06 & 0.06 \\
\hline CRIS-134 × S-12 & 0.14 & 0.14 & 0.08 & 0.13 & 0.03 \\
\hline CIM-446 × S-12 & 0.48 & 0.20 & 0.11 & -0.02 & 0.05 \\
\hline FH-900 × S-12 & 0.70 & -0.08 & 0.06 & -0.12 & 0.02 \\
\hline MNH-93 × S-12 & 1.13 & 0.98 & 0.06 & 0.08 & -0.02 \\
\hline CIM-707 × S-12 & 0.54 & -3.00 & -0.07 & 0.07 & 0.04 \\
\hline CIM-482 × S-12 & 0.69 & 1.89 & -0.13 & 0.12 & -0.01 \\
\hline NIAB-111 $\times$ ACALA-1517-C & 0.75 & -1.23 & 0.05 & 0.13 & -0.02 \\
\hline CP-15/2 $\times$ ACALA-1517-C & 0.04 & -1.43 & 0.01 & 0.07 & 0.07 \\
\hline
\end{tabular}




\begin{tabular}{lccccc}
\hline BH-160 × ACALA-1517-C & 0.51 & 0.83 & 0.08 & -0.01 & 0.07 \\
CIM-1100 × ACALA-1517-C & -0.10 & 1.21 & -0.09 & -0.04 & -0.01 \\
CRIS-134 × ACALA-1517-C & -0.18 & 0.62 & 0.04 & -0.01 & 0.06 \\
CIM-446 × ACALA-1517-C & 0.05 & 2.01 & -0.06 & 0.01 & 0.04 \\
FH-900 × ACALA-1517-C & -0.12 & -1.89 & -0.13 & 0.01 & -0.05 \\
MNH-93 × ACALA-1517-C & -0.40 & -0.54 & 0.01 & -0.21 & -0.05 \\
CIM-707 × ACALA-1517-C & -0.38 & 0.41 & 0.11 & 0.01 & -0.07 \\
CIM-482 × ACALA-1517-C & -0.16 & 0.01 & 0.03 & 0.05 & -0.04 \\
Standard Error & 12.89 & 11.45 & 6.57 & 0.36 & 3.84 \\
\hline
\end{tabular}

Table 5. Specific combining ability estimates of various quantitative traits of cotton grown under water stress conditions.

\begin{tabular}{|c|c|c|c|c|c|}
\hline Crosses & SC & TR & CHL $a$ & CHL $b$ & CAR \\
\hline NIAB-111 × CIM-506 & -0.18 & 0.80 & 0.03 & -0.14 & -0.04 \\
\hline CP-15/2 × CIM-506 & 0.06 & 1.04 & 0.10 & 0.03 & 0.01 \\
\hline BH-160 × CIM-506 & 0.14 & 0.91 & -0.13 & -0.05 & -0.03 \\
\hline CIM-1100 × CIM-506 & -0.19 & 0.69 & 0.06 & -0.04 & -0.01 \\
\hline CRIS-134 × CIM-506 & -0.09 & -0.69 & -0.24 & 0.01 & 0.18 \\
\hline CIM-446 × CIM-506 & 0.25 & -1.56 & -0.13 & 0.02 & -0.09 \\
\hline FH-900 × CIM-506 & -0.02 & -0.25 & 0.06 & 0.07 & 0.02 \\
\hline MNH-93 × CIM-506 & -0.03 & -0.20 & -0.06 & 0.05 & 0.01 \\
\hline CIM-707 × CIM-506 & 0.19 & 0.06 & 0.12 & 0.09 & 0.02 \\
\hline CIM-482 × CIM-506 & -0.13 & -0.79 & 0.19 & -0.05 & -0.08 \\
\hline NIAB-111 $\times$ NIAB Karishma & 0.13 & 1.16 & -0.14 & 0.76 & -0.05 \\
\hline CP-15/2 × NIAB Karishma & -0.84 & 0.77 & 0.06 & -0.14 & -0.05 \\
\hline BH-160 × NIAB Karishma & -0.42 & 0.74 & -0.05 & -0.05 & -0.05 \\
\hline CIM-1100 × NIAB Karishma & -0.16 & -1.12 & -0.04 & 0.54 & 0.03 \\
\hline CRIS-134 × NIAB Karishma & 0.04 & -0.70 & -0.05 & 0.04 & 0.05 \\
\hline CIM-446 × NIAB Karishma & 0.19 & -0.24 & 0.10 & 0.03 & 0.14 \\
\hline FH-900 × NIAB Karishma & 0.12 & 0.01 & 0.05 & 0.11 & 0.01 \\
\hline MNH-93 $\times$ NIAB Karishma & -0.12 & -0.11 & 0.10 & -0.02 & 0.02 \\
\hline CIM-707 × NIAB Karishma & 0.26 & -0.05 & 0.12 & 0.01 & -0.06 \\
\hline CIM-482 × NIAB Karishma & 0.78 & -0.46 & -0.15 & 0.04 & 0.03 \\
\hline NIAB-111 × MNH-129 & 0.19 & -1.52 & 0.10 & 0.12 & 0.04 \\
\hline CP-15/2 × MNH-129 & 0.22 & 0.22 & 0.08 & -0.02 & 0.08 \\
\hline BH-160 × MNH-129 & 0.11 & -0.17 & 0.09 & 1.23 & 0.03 \\
\hline CIM-1100 × MNH-129 & -0.13 & 0.50 & 0.08 & 0.05 & -0.04 \\
\hline CRIS-134 × MNH-129 & 0.34 & 0.79 & 0.06 & -0.04 & -0.07 \\
\hline CIM-446 × MNH-129 & 0.15 & 0.58 & -0.07 & 0.04 & -0.05 \\
\hline FH-900 × MNH-129 & -0.18 & 0.23 & -0.04 & -0.12 & -0.03 \\
\hline MNH-93 × MNH-129 & 0.21 & -0.56 & -0.15 & 0.04 & -0.01 \\
\hline CIM-707 × MNH-129 & -0.58 & -0.06 & -0.19 & -0.11 & 0.04 \\
\hline CIM-482 × MNH-129 & -0.36 & -0.01 & 0.03 & 0.03 & 0.04 \\
\hline NIAB-111 $\times$ FH-1000 & -0.08 & -0.86 & -0.04 & 0.02 & 0.01 \\
\hline CP-15/2 × FH-1000 & 0.25 & -1.05 & -0.07 & 0.19 & -0.04 \\
\hline BH-160 × FH-1000 & 0.31 & -0.48 & 0.02 & 0.08 & 0.02 \\
\hline CIM-1100 × FH-1000 & -0.06 & 0.10 & -0.11 & 0.07 & -0.04 \\
\hline CRIS-134 × FH-1000 & -0.13 & 1.01 & -0.13 & -0.12 & -0.09 \\
\hline CIM-446 × FH-1000 & -0.12 & 0.61 & 0.02 & -0.08 & -0.01 \\
\hline FH-900 × FH-1000 & 0.32 & 0.59 & 0.26 & -0.01 & -0.03 \\
\hline MNH-93 $\times$ FH-1000 & -0.26 & -0.13 & 0.10 & 0.10 & 0.03 \\
\hline CIM-707 × FH-1000 & 0.02 & -0.34 & -0.04 & -0.04 & 0.09 \\
\hline CIM-482 × FH-1000 & -0.26 & 0.55 & -0.01 & -0.11 & 0.07 \\
\hline NIAB-111 × S-12 & -0.10 & -0.30 & 0.04 & -0.15 & 0.03 \\
\hline $\mathrm{CP}-15 / 2 \times \mathrm{S}-12$ & 0.03 & -0.89 & -0.14 & -0.10 & -0.03 \\
\hline
\end{tabular}




\begin{tabular}{|c|c|c|c|c|c|}
\hline BH-160 × S-12 & 0.12 & -0.99 & 0.05 & 0.06 & 0.03 \\
\hline CIM-1100 × S-12 & 0.42 & 0.02 & -0.05 & 0.01 & 0.05 \\
\hline CRIS-134 × S-12 & -0.08 & -0.43 & 0.02 & 0.08 & -0.02 \\
\hline CIM-446 × S-12 & -0.64 & 0.80 & 0.28 & -0.03 & 0.01 \\
\hline FH-900 × S-12 & 0.10 & -0.49 & -0.04 & -0.04 & 0.01 \\
\hline MNH-93 × S-12 & -0.01 & 1.36 & 0.06 & 0.03 & 0.05 \\
\hline CIM-707 × S-12 & 0.33 & 0.46 & -0.06 & 0.05 & -0.01 \\
\hline CIM-482 × S-12 & -0.18 & 0.45 & -0.15 & 0.10 & -0.01 \\
\hline NIAB-111 $\times$ ACALA-1517-C & 0.03 & 0.74 & 0.01 & 0.15 & 0.03 \\
\hline CP-15/2 × ACALA-1517-C & 0.27 & -0.09 & -0.02 & 0.04 & 0.02 \\
\hline BH-160 × ACALA-1517-C & -0.28 & -0.01 & 0.03 & -0.04 & 0.05 \\
\hline CIM-1100 × ACALA-1517-C & 0.12 & -0.20 & 0.06 & -0.08 & 0.06 \\
\hline CRIS-134 × ACALA-1517-C & -0.08 & 0.01 & 0.33 & 0.02 & 0.01 \\
\hline CIM-446 × ACALA-1517-C & 0.16 & -0.19 & -0.20 & 0.02 & 0.02 \\
\hline FH-900 × ACALA-1517-C & -0.34 & -0.08 & -0.29 & -0.01 & 0.02 \\
\hline MNH-93 $\times$ ACALA-1517-C & 0.19 & -0.36 & -0.05 & -0.09 & -0.05 \\
\hline CIM-707 $\times$ ACALA-1517-C & -0.23 & -0.07 & 0.06 & 0.01 & -0.09 \\
\hline CIM-482 × ACALA-1517-C & 0.15 & 0.25 & 0.09 & -0.01 & 0.02 \\
\hline Standard Error & 4.55 & 10.64 & 3.69 & 5.89 & 0.95 \\
\hline
\end{tabular}

Table 6. Heterosis and Heterobeltiosis estimates of various quantitative traits of cotton grown under normal water conditions.

\begin{tabular}{|c|c|c|c|c|c|c|c|c|c|c|}
\hline \multirow[t]{2}{*}{ Crosses } & \multicolumn{5}{|c|}{ Heterosis } & \multicolumn{5}{|c|}{ Heterobeltiosis } \\
\hline & $\mathrm{SC}$ & TR & CHL $a$ & CHL $b$ & CAR & $\mathrm{SC}$ & TR & CHL $a$ & CHL $b$ & CAR \\
\hline NIAB-111 × CIM-506 & -14.39 & -3.09 & -1.85 & -3.68 & -8.99 & -23.27 & -15.32 & -2.62 & -4.27 & -16.06 \\
\hline CP-15/2 × CIM-506 & 8.83 & 0.71 & -6.2 & -2.82 & 5.14 & -1.91 & -16.86 & -11.88 & -5.49 & 3.57 \\
\hline BH-160 × CIM-506 & 12.37 & -45.54 & -12.59 & -4.43 & 2.21 & -2.89 & -56.74 & -16.41 & -7.93 & -7.04 \\
\hline CIM-1100 × CIM-506 & 3.14 & -33.64 & -2.3 & -5.4 & 21.34 & -8.07 & -45.8 & -2.36 & -10.98 & 20.61 \\
\hline CRIS-134 × CIM-506 & -41.75 & -6.81 & -10.54 & -5.35 & 14.1 & -47.8 & -14.93 & -10.72 & -10.16 & 6.75 \\
\hline CIM-446 × CIM-506 & -41.04 & -19.92 & -16.55 & 5.92 & 6.23 & -44.37 & -38.59 & -20.38 & -3.66 & -11.04 \\
\hline FH-900 × CIM-506 & -24.16 & 68.82 & 0.85 & 8.27 & 23.18 & -28.67 & 52.43 & 0.13 & 0.57 & 14.11 \\
\hline MNH-93 × CIM-506 & -38.81 & 2.14 & -5.04 & 7.45 & 28.85 & -42.25 & -12.85 & -9.7 & 1.02 & 23.31 \\
\hline CIM-707 × CIM-506 & -33.33 & -24.35 & -0.32 & 9.01 & 4.45 & -38.1 & -37.74 & -2.27 & -1.63 & 1.15 \\
\hline CIM-482 × CIM-506 & -39.64 & 0.66 & -1.37 & -8.61 & -20.22 & -44.3 & -20.76 & -6.16 & -12.6 & -26.42 \\
\hline NIAB-111 × NIAB KARISHMA & -16.72 & 44.11 & -5.82 & -17.6 & -6.84 & -24.84 & 18.47 & -8.54 & -19.55 & -14.56 \\
\hline CP-15/2 × NIAB KARISHMA & -9.47 & 43.22 & -5.23 & -16.59 & -4.56 & -17.83 & 11.76 & -9.11 & -16.77 & -6.55 \\
\hline BH-160 × NIAB KARISHMA & -45.52 & 24.14 & -8.15 & -10.77 & -22.78 & -52.6 & -6.45 & -10.3 & -11.45 & -30.15 \\
\hline CIM-1100 × NIAB KARISHMA & -39.79 & 39.21 & 2.27 & -7.69 & -0.61 & -45.96 & 7.6 & 0.15 & -10.58 & -1.82 \\
\hline CRIS-134 × NIAB KARISHMA & -21.95 & 79.65 & -0.58 & -7.62 & 11.55 & -29.56 & 53.73 & -2.51 & -9.72 & 4.97 \\
\hline CIM-446 × NIAB KARISHMA & -20.74 & -24.23 & -2.63 & 9.47 & 4.8 & -24.65 & -44.69 & -5.13 & 2.38 & -11.8 \\
\hline FH-900 × NIAB KARISHMA & -21.03 & 62.75 & 5.04 & 12.54 & 22.67 & -25.17 & 37.86 & 3.52 & 7.56 & 14.29 \\
\hline MNH-93 × NIAB KARISHMA & -28.15 & -24.87 & 1.65 & -8.49 & 29.03 & -31.69 & -39.57 & -1.3 & -11.45 & 24.22 \\
\hline CIM-707 × NIAB KARISHMA & -19.27 & 54 & 2.96 & 7.8 & -15.22 & -24.49 & 19.84 & 2.76 & 0 & -18.39 \\
\hline CIM-482 × NIAB KARISHMA & -11.19 & 5.56 & -9.02 & 0.44 & 7.91 & -17.45 & -21.11 & -11.61 & -1.08 & -1.04 \\
\hline NIAB-111 × MNH-129 & -7.19 & 20.33 & -2.14 & 0.65 & 8.33 & -18.87 & -1.35 & -4.68 & -4.53 & -5.7 \\
\hline CP-15/2 × MNH-129 & 11.59 & -0.76 & -10.01 & -10.1 & 21.54 & -1.91 & -22.75 & -13.96 & -12.9 & 12.5 \\
\hline BH-160 × MNH-129 & 3.42 & -16.98 & -7.26 & -0.67 & -6.9 & -12.72 & -37.59 & -9.7 & -2.85 & -20 \\
\hline CIM-1100 $\times$ MNH-129 & 11.43 & -18.81 & -4.5 & -4.37 & 5.84 & -3.11 & -37.4 & -6.19 & -4.59 & -1.21 \\
\hline CRIS-134 × MNH-129 & -0.72 & 7.29 & -1.8 & -7.54 & -6.81 & -13.21 & -8.46 & -3.41 & -8.17 & -7.13 \\
\hline CIM-446 × MNH-129 & -15.71 & 12.58 & -14.36 & 7.03 & 21.74 & -22.54 & -18.01 & -16.81 & 2.98 & 7.69 \\
\hline FH-900 × MNH-129 & -29.77 & 54.6 & -4.22 & -5.13 & 12.77 & -35.66 & 30.58 & -5.31 & -6.65 & 11.19 \\
\hline MNH-93 × MNH-129 & -21.84 & 50.66 & -12.35 & -0.36 & -4.79 & -28.17 & 20.85 & -15.15 & -0.69 & -6.71 \\
\hline CIM-707 × MNH-129 & -11.28 & 55.39 & -14.65 & -5.41 & 9.15 & -19.73 & 20.62 & -14.75 & -9.75 & -0.57 \\
\hline CIM-482 × MNH-129 & -32.84 & -5.34 & -7.65 & -0.57 & -0.6 & -39.6 & -29.41 & -10.55 & -2 & -13.47 \\
\hline NIAB-111 × FH-1000 & -31.6 & -3.3 & -1.82 & -14.96 & 24.07 & -42.14 & -20.72 & -4.07 & -15.23 & -1.97 \\
\hline CP-15/2 × FH-1000 & -23.6 & 46.6 & -10.22 & 4.22 & 14.29 & -35.03 & 14.12 & -14.42 & 2.28 & -4.76 \\
\hline BH-160 × FH-1000 & -27.92 & 22.64 & -4.5 & 3.73 & 7.4 & -41.04 & -7.8 & -7.31 & 0.83 & -16.08 \\
\hline CIM-1100 $\times$ FH-1000 & -30.63 & 40.59 & -9.23 & 0.76 & 25.63 & -41.61 & 8.4 & -10.56 & -4.35 & 5.45 \\
\hline
\end{tabular}




\begin{tabular}{|c|c|c|c|c|c|c|c|c|c|c|}
\hline CRIS-134 × FH-1000 & -17.47 & 50.44 & -3.68 & -13.51 & 22.83 & -30.19 & 28.36 & -4.96 & -17.18 & 9.86 \\
\hline CIM-446 × FH-1000 & -3.17 & 8.08 & -14.95 & 3.61 & 54.96 & -14.08 & -21.29 & -17.64 & -4.97 & 53.57 \\
\hline FH-900 × FH-1000 & -7.51 & 52.18 & -12.51 & 6.96 & 35.46 & -18.18 & 28.54 & -13.23 & 0.21 & 22.3 \\
\hline MNH-93 × FH-1000 & -19.84 & 64.99 & -3.74 & -2.85 & 42.53 & -28.87 & 32.34 & -7.1 & -7.87 & 24.83 \\
\hline CIM-707 × FH-1000 & -27.63 & 42.56 & -3.1 & -3.75 & 42.66 & -36.73 & 10.66 & -3.53 & -12.42 & 17.24 \\
\hline CIM-482 × FH-1000 & -19.69 & 16.94 & -3.93 & -16.31 & 28.52 & -30.2 & -12.8 & -7.23 & -19.25 & 1.55 \\
\hline NIAB-111 × S-12 & -33.8 & 4.17 & 5.88 & -22.22 & -12.4 & -40.88 & -9.91 & 5.6 & -22.22 & -13.99 \\
\hline CP-15/2 × S-12 & -36.17 & -5.04 & -9.73 & -13.56 & -17.51 & -42.68 & -22.35 & -16.03 & -15.43 & -21.51 \\
\hline BH-160 × S-12 & -35.57 & 16.67 & -0.32 & -2.97 & -36.1 & -44.51 & -8.16 & -5.63 & -5.97 & -38.19 \\
\hline CIM-1100 × S-12 & -23.78 & 32.55 & -0.73 & 0.22 & 5.98 & -32.3 & 7.25 & -1.83 & -5.14 & 0 \\
\hline CRIS-134 × S-12 & -15.49 & 41.6 & 5.52 & 2.59 & 1.22 & -24.53 & 27.86 & 4.21 & -2.06 & -10.75 \\
\hline CIM-446 × S-12 & -4.12 & 7.82 & -2.08 & 5.51 & 12.84 & -9.86 & -18.01 & -7.51 & -3.5 & -10.22 \\
\hline FH-900 × S-12 & 0.75 & 53.53 & 4.81 & -3.74 & 5.23 & -5.59 & 37.14 & 2.98 & -10.08 & -8.06 \\
\hline MNH-93 × S-12 & 4.87 & 43.58 & 3.58 & 2.06 & -3.28 & -1.41 & 21.28 & -2.49 & -3.5 & -12.9 \\
\hline CIM-707 × S-12 & -7.35 & -17.42 & -0.71 & 8.16 & -10.56 & -14.29 & -32.68 & -3.66 & -1.85 & -13.44 \\
\hline CIM-482 × S-12 & -5.11 & 40.13 & -6.16 & 4.39 & -17.15 & -12.75 & 9.34 & -11.61 & 0.41 & -18.65 \\
\hline NIAB-111 × ACALA-1517-C & -7.58 & -4.96 & 0.27 & 6.23 & -1.65 & -19.5 & -18.02 & 0 & 0 & -7.25 \\
\hline CP-15/2 × ACALA-1517-C & -14.91 & -2.88 & -8.45 & 5.82 & 19.17 & -25.48 & -20.78 & -14.42 & 1.72 & 18.13 \\
\hline BH-160 × ACALA-1517-C & -12.03 & 18.74 & -3.84 & 4.18 & 3.24 & -26.01 & -6.74 & -8.5 & 1.1 & -4.02 \\
\hline CIM-1100 $\times$ ACALA-1517-C & -24.73 & 37.59 & -7.98 & 1.27 & 10.71 & -34.78 & 11.07 & -8.52 & 0.69 & 8.77 \\
\hline CRIS-134 × ACALA-1517-C & -30.69 & 51.93 & -3.09 & 0.8 & 22.68 & -39.62 & 36.82 & -3.79 & -0.68 & 12.28 \\
\hline CIM-446 × ACALA-1517-C & -22.31 & 32.63 & -13.87 & 15.87 & 30.25 & -28.87 & 0.64 & -18.24 & 12.35 & 7.02 \\
\hline FH-900 × ACALA-1517-C & -26.44 & 26.43 & -8.06 & 13.28 & 7.74 & -32.87 & 12.62 & -9.19 & 12.35 & -2.34 \\
\hline MNH-93 $\times$ ACALA-1517-C & -38.46 & 22.73 & -3.44 & -9.52 & 6.88 & -43.66 & 3.4 & -8.64 & -9.95 & 0 \\
\hline CIM-707 × ACALA-1517-C & -36.6 & 33.49 & 0.97 & 12.73 & -8.99 & -42.86 & 8.56 & -1.51 & 8.39 & -9.77 \\
\hline CIM-482 $\times$ ACALA-1517-C & -32.58 & 16.89 & -5.26 & 8.2 & -7.69 & -39.6 & -9 & -10.31 & 5.79 & -12.95 \\
\hline
\end{tabular}

Table 7. Heterosis and Heterobeltiosis estimates of various quantitative traits of cotton grown under water stress conditions.

\begin{tabular}{|c|c|c|c|c|c|c|c|c|c|c|}
\hline \multirow[t]{2}{*}{ Crosses } & \multicolumn{5}{|c|}{ Heterosis } & \multicolumn{5}{|c|}{ Heterobeltiosis } \\
\hline & SC & TR & CHL $a$ & CHL $b$ & CAR & $\mathrm{SC}$ & TR & CHL $a$ & CHL $b$ & CAR \\
\hline NIAB-111 × CIM-506 & -17.78 & -2.51 & -14.34 & -15.82 & -0.97 & -22.92 & -2.88 & -22.46 & -18.58 & -0.97 \\
\hline CP-15/2 × CIM-506 & -13.04 & 7.17 & -12.62 & 4.51 & 25.97 & -16.67 & 2.61 & -22.67 & 4.06 & 10.68 \\
\hline BH-160 × CIM-506 & -7.69 & 7.64 & -20.16 & 5.19 & -10.38 & -14.29 & 0.62 & -25.61 & 3.8 & -12.84 \\
\hline CIM-1100 × CIM-506 & -22.73 & -4.83 & -5.02 & 4.88 & 23.6 & -26.09 & -8 & -6.32 & 0.58 & 6.8 \\
\hline CRIS-134 × CIM-506 & -3.8 & -44.9 & -16.78 & 6.51 & 117.5 & -9.52 & -47.4 & -17.32 & 2.92 & 68.93 \\
\hline CIM-446 × CIM-506 & -10 & -53.68 & -21.37 & 17.25 & -7.1 & -15.63 & -54.48 & -26.42 & 12.28 & -17.48 \\
\hline FH-900 × CIM-506 & -6.52 & -29.89 & 6.07 & 16.74 & -4.13 & -9.52 & -32.14 & 5.84 & 16.23 & -16.55 \\
\hline MNH-93 × CIM-506 & -4.54 & -24.16 & -10.63 & 9.49 & 21.98 & -7.38 & -27.14 & -17.43 & 9.33 & 7.77 \\
\hline CIM-707 × CIM-506 & -15.73 & -28.92 & 0.52 & 15.22 & 11.54 & -20.21 & -30.61 & -2.64 & 12.87 & 10.48 \\
\hline CIM-482 × CIM-506 & -5 & -51.89 & 1.39 & 4.57 & -19.21 & -9.52 & -53.64 & -6.25 & 0.29 & -20.39 \\
\hline NIAB-111 $\times$ NIAB KARISHMA & -4.88 & 13.62 & -19.7 & -8.5 & -9.8 & -18.75 & 5.04 & -24.91 & -9.6 & -10.68 \\
\hline CP-15/2 × NIAB KARISHMA & -47.59 & 9.23 & -10.95 & -15.83 & -1.68 & -50.65 & -3.27 & -18.67 & -19.2 & -12.87 \\
\hline BH-160 × NIAB KARISHMA & -28.57 & 11.83 & -10.96 & -1.13 & -24.76 & -30.56 & -3.11 & -14.21 & -6.67 & -27.52 \\
\hline CIM-1100 $\times$ NIAB KARISHMA & -20 & -38.06 & -6.49 & 2.76 & 27.27 & -30.43 & -44.67 & -8.47 & -5.6 & 10.89 \\
\hline CRIS-134 × NIAB KARISHMA & 5.63 & -41.18 & 0 & 3.17 & 37.98 & 1.35 & -48.05 & -4.03 & -4.53 & 7.92 \\
\hline CIM-446 × NIAB KARISHMA & -9.76 & -20.15 & -3.31 & 11.63 & 56.91 & -22.92 & -27.59 & -6.42 & 2.4 & 40.59 \\
\hline FH-900 × NIAB KARISHMA & 2.32 & -18.07 & 9.62 & 13.33 & -13.33 & -4.58 & -22.14 & 5.65 & 8.8 & -25.18 \\
\hline MNH-93 × NIAB KARISHMA & -4.76 & -15.79 & 2.59 & -2.51 & 18.89 & -11.39 & -19.38 & -2.02 & -6.67 & 5.94 \\
\hline CIM-707 × NIAB KARISHMA & -11.11 & -26.04 & 4.75 & 2.42 & -20.39 & -23.4 & -33.33 & 4.44 & -4 & -21.9 \\
\hline CIM-482 × NIAB KARISHMA & 36.11 & -41.26 & -14.23 & 5.95 & -2.49 & 28.95 & -47.68 & -18.01 & -2.67 & -2.97 \\
\hline NIAB-111 × MNH-129 & -5.68 & -42.64 & -13.44 & 7.02 & 8.6 & -13.54 & -45.32 & -20.35 & 4.1 & -1.94 \\
\hline CP-15/2 × MNH-129 & -7.01 & 1.79 & -17.33 & -0.14 & 35.4 & -8.75 & -7.19 & -25.67 & -0.29 & 31.33 \\
\hline BH-160 × MNH-129 & -9.21 & -3.14 & -10.65 & 9.87 & -12.5 & -13.75 & -13.66 & -15.33 & 7.8 & -22.94 \\
\hline CIM-1100 $\times$ MNH-129 & -20.93 & 2.9 & -7.34 & 13.94 & -7.6 & -26.09 & -5.33 & -7.72 & 8.67 & -12.05 \\
\hline CRIS-134 × MNH-129 & 12.99 & -3.57 & -1.39 & 2.26 & 2.86 & 8.75 & -12.34 & -3.76 & -1.73 & -13.25 \\
\hline CIM-446 × MNH-129 & -13.64 & 3.32 & -21.11 & 18.97 & -14.11 & -20.83 & -3.45 & -24.91 & 13.29 & -15.66 \\
\hline FH-900 × MNH-129 & -12.99 & -7.39 & -4.37 & 0.43 & -32.43 & -13.75 & -9.16 & -6.26 & 0.29 & -46.04 \\
\hline MNH-93 × MNH-129 & 4.4 & -20.78 & -19.14 & 8.27 & -2.47 & 3.75 & -21.71 & -24.04 & 7.8 & -4.82 \\
\hline CIM-707 $\times$ MNH-129 & -42.53 & -20.88 & -22.22 & -2.08 & -1.06 & -46.81 & -26.53 & -23.33 & -4.62 & -11.43 \\
\hline
\end{tabular}




\begin{tabular}{|c|c|c|c|c|c|c|c|c|c|c|}
\hline CIM-482 × MNH-129 & -14.1 & -25.63 & -11.63 & 12.12 & -12.57 & -16.25 & -31.79 & -16.91 & 6.94 & -20 \\
\hline NIAB-111 × FH-1000 & -3.85 & -40.94 & -13.71 & -2.09 & 3.78 & -21.88 & -46.04 & -20.53 & -3.83 & -6.8 \\
\hline CP-15/2 × FH-1000 & 8.03 & -38.06 & -18.15 & 17.77 & -6.25 & -3.9 & -45.75 & -26.33 & 16.43 & -8.54 \\
\hline BH-160 × FH-1000 & 13.64 & -21.01 & -6.6 & 15.74 & -15.18 & 4.17 & -32.3 & -11.4 & 12.46 & -25.69 \\
\hline CIM-1100 × FH-1000 & -7.89 & -17.74 & -10.79 & 14.54 & -1.91 & -23.91 & -27.33 & -11.25 & 8.22 & -6.1 \\
\hline CRIS-134 × FH-1000 & 8.96 & -10.04 & -4.49 & -4.76 & -0.72 & -1.35 & -21.43 & -6.88 & -9.35 & -15.85 \\
\hline CIM-446 × FH-1000 & -12.82 & -7.69 & -7.92 & 8.11 & 4.94 & -29.17 & -17.24 & -12.26 & 1.98 & 3.66 \\
\hline FH-900 × FH-1000 & 21.21 & -11.38 & 23.4 & 8.88 & -28.51 & 6.87 & -16.79 & 20.83 & 7.65 & -43.17 \\
\hline MNH-93 × FH-1000 & -0.72 & -23.77 & 3.22 & 4.89 & 19.26 & -12.66 & -27.91 & -2.94 & 3.4 & 17.07 \\
\hline CIM-707 × FH-1000 & -11.69 & -39.69 & -4.42 & 3.67 & 21.93 & -27.66 & -46.26 & -5.68 & 0 & 8.57 \\
\hline CIM-482 × FH-1000 & 2.94 & -25.56 & -5.66 & -1.05 & 16.48 & -7.89 & -34.44 & -11.21 & -6.52 & 6 \\
\hline NIAB-111 × S-12 & -15.79 & -29.06 & -4.74 & -16.91 & -27.85 & -25 & -32.37 & -15.44 & -17.69 & -31.9 \\
\hline CP-15/2 × S-12 & -14.47 & -35.48 & -18.04 & -8.08 & -30.93 & -15.58 & -41.18 & -28.83 & -11.53 & -42.24 \\
\hline BH-160 × S-12 & -8.84 & -33.1 & -0.1 & 12.46 & -42.22 & -10.67 & -40.37 & -8.79 & 6.43 & -43.97 \\
\hline CIM-1100 × S-12 & -1.8 & -21.01 & -2.07 & 8.01 & -4.71 & -10.87 & -27.33 & -5.47 & -0.54 & -21.55 \\
\hline CRIS-134 × S-12 & -3.36 & -42.86 & 10.74 & 11.56 & -12.14 & -4 & -48.05 & 9.04 & 3.49 & -34.48 \\
\hline CIM-446 × S-12 & -41.52 & -5.54 & 12.96 & 10.5 & -21.43 & -47.92 & -11.72 & 3.58 & 1.61 & -33.62 \\
\hline FH-900 × S-12 & -2.34 & -38.52 & 9.98 & 5.01 & -38.82 & -4.58 & -39.69 & 7.83 & 1.07 & -43.88 \\
\hline MNH-93 × S-12 & -3.9 & 9.8 & 6.18 & 5.87 & -25.13 & -6.33 & 8.53 & -3.85 & 1.61 & -37.07 \\
\hline CIM-707 × S-12 & -11.24 & -23.08 & -0.75 & 10.41 & -36.65 & -20.21 & -28.57 & -5.88 & 3.75 & -39.66 \\
\hline CIM- $482 \times$ S-12 & -7.28 & -29.24 & -9.53 & 15.87 & -37.96 & -7.89 & -35.1 & -18.01 & 6.7 & -42.24 \\
\hline NIAB-111 × ACALA-1517-C & -15.29 & -3.4 & -11.33 & 25.16 & 1.87 & -25 & -7.91 & -20.35 & 8.74 & -1.8 \\
\hline CP-15/2 × ACALA-1517-C & -9.93 & -16.13 & -15.56 & 21.63 & 7.94 & -11.69 & -23.53 & -25.83 & 8.41 & -8.11 \\
\hline BH-160 × ACALA-1517-C & -30.14 & -10.8 & -6.17 & 22.72 & -8.18 & -31.08 & -20.5 & -13.27 & 11.11 & -9.01 \\
\hline CIM-1100 $\times$ ACALA-1517-C & -16.87 & -23.91 & 0.32 & 18.15 & 3.33 & -25 & -30 & -1.89 & 9.87 & -13.42 \\
\hline CRIS-134 × ACALA-1517-C & -8.11 & -31.43 & 25.27 & 25.3 & 21.43 & -8.11 & -37.66 & 25 & 15.67 & -8.11 \\
\hline CIM-446 × ACALA-1517-C & -17.65 & -25.46 & -21.54 & 35.51 & -2.62 & -27.08 & -30.34 & -27.17 & 26.2 & -16.22 \\
\hline FH-900 × ACALA-1517-C & -23.98 & -26.85 & -12.04 & 26.18 & -20 & -26.21 & -28.24 & -12.61 & 12.46 & -28.06 \\
\hline MNH-93 $\times$ ACALA-1517-C & -0.65 & -28.63 & -5.51 & 12.23 & -18.95 & -3.8 & -29.46 & -13.39 & 0.29 & -30.63 \\
\hline CIM-707 × ACALA-1517-C & -35.71 & -32.6 & 1.58 & 25.42 & -37.96 & -42.55 & -37.42 & -2.43 & 14.33 & -39.64 \\
\hline CIM-482 $\times$ ACALA-1517-C & 1.33 & -31.41 & 0 & 25.34 & -8.06 & 0 & -37.09 & -8.27 & 16.56 & -12.61 \\
\hline
\end{tabular}

\section{DISCUSSION}

Water stress seriously affects cotton as well as other field crops productivity around the globe and this type of stress has remained a major challenge to plant biologists and researchers (Davis et al., 2017; Khan et al., 2017). Economic survey of Pakistan (2016-17) also indicated that water stress at various critical stages is one of the reasons of low productivity in field crops, and these increasing losses warn the development of new germplasm keeping in view the adverse effects of climate changes (Govt. of Pakistan, 2016-17). Under water stress, chlorophyll contents i.e. $a$ and $b$ are reduced in Triticum aestivum L. (Arshad et al., 2016), likewise photosynthetic activity is also significantly affected due to water stress because this stress impaired with photosynthetic machinery. In continuation, photosynthetic pigments i.e. carotenoids are decreased in Zea mays L. and Hordeum vulgare L. (Ghahfarokhi et al., 2015; Arivalagan and Somasundaram, 2016). The contents of chlorophyll $a, b$ and carotenoids were decreased in the leaves of water stress susceptible genotypes (El-Tayeb, 2006). Similar findings are reported from current study that under water stress, reduction in chlorophyll $a, b$ and total carotenoids were observed. Carotenoids is considered as powerful reactive oxygen species (ROS) scavengers (Edreva 2005a and b; Leopoldidni et al., 2006) and abiotic stresses like water stress stimulates the production of different secondary metabolites (Wahid and Ghazanfar, 2006). The water stress-tolerant cultivar had higher content of chlorophyll $a, b$ and carotene than the sensitive cultivar (Shah et al., 2011). Variability in net photosynthesis and stomatal conductance have been suggested as tools for screening of cotton germplasm (Yu et al., 2016). Because water stress leads to significant reduction in net photosynthesis in response to stomatal closure, which restricts the diffusion of $\mathrm{CO}_{2}$ into the leaf (Osório et al., 2011). Transpiration rate decreases with a decreased level of moisture. The reason behind decline in moisture stress might be because of lowered water potentials in the root zone, it triggers a signal from root to shoot (He et al., 2005).

The molecular mechanism revealed that genes during water-stress conditions are thought to function not only in protecting cells from water deficit by the production of important metabolic proteins but also in the regulation of genes for signal transduction in the waterstress response. Increase of gene expression is also positively associated with tolerance to water stress (Xiong et al., 2010). In addition, genetic analysis was partitioned variations into various genetic components. Variance due to GCA was lower than variance due to specific combing ability for transpiration rate, stomatal conductance, carotenoids chlorophyll $a$, and chlorophyll 
$b$ which indicated the existence of non-additive genes (Javaid et al., 2014). Higher magnitude of SCA variance for studied traits is supported by Munir et al. (2018) and indicate the importance of non-additive genes in expression controlling the traits in upland cotton. The findings of Shakoor et al. (2010) also supported the role of non-additive factors in the inheritance of traits mentioned. In addition, water stress also reduces the activity of photosynthesis associated physiological markers i.e. stomatal conductance and transpiration rate (Han et al., 2016). These markers were also exploited in segregating population, and higher estimates of stomatal conductance examined in combination of CIM-482 $\times$ NIAB Karishma with low $\times$ high GCA. Cross MNH-93 $\times$ $\mathrm{S}-12$ proved as good combination for transpiration rate due to hybridization of low $\times$ low general combiners. For chlorophyll a CRIS-134 $\times$ ACALA-1517-C exhibited good performance by involving high $\times$ low combiners, whilst CP-15/2 $\times$ FH-1000 identified as a good combination for chlorophyll $b$ due to low $\times$ low parents and SCA effects for high carotenoids of CRIS-134 $\times$ CIM-506 due to high $\times$ high GCA estimates. Specific combining ability effects responsible for dominant gene action (Aslam et al., 2017). These estimates indicated the potential of these combinations for the development new genotypes suitable for water stress conditions.

Conclusion: This study revealed a considerable genetic diversity in studied germplasm. Identified superior hybrid combinations (CIM-482 $\times$ NIAB Karishma for stomatal conductance and carotenoids, MNH-93 $\times \mathrm{S}-12$ for transpiration rate, CRIS-134 $\times$ ACALA-1517-C for chlorophyll a, CP-15/2 $\times$ FH-1000 for chlorophyll $b$ and CRIS-134 $\times$ CIM-506 for carotenoids) may be used in breeding programs to develop drought tolerant cotton variety. Non-additive type of genetic control predict that selection must be delayed till later generations for all desirable traits. As study was conducted under field conditions of subtropical semiarid regions therefore, these results may be helpful for breeders working under such climatic conditions.

\section{REFERENCES}

Abid, M., A. Hakeem, Y. Shao, Y. Liu, R. Zahoor, Y. Fan, J. Suyu, S.T. Ata-Ul-Karim, Z. Tian and D. Jiang (2018). Seed osmopriming invokes stress memory against post-germinative drought stress in wheat (Triticum aestivum L.). Environ. Exper. Bot. 145:12-20.

Arivalagan, M. and R.G. Somasundaram (2016). Effect of propiconazole and salicylic acid on the growth and photosynthetic pigments in Sorghum bicolor (L.) Moench. under drought condition. J. Ecobiotechn. 7: 17-23.
Arshad, M., S. Ali, A. Noman, Q. Ali, M. Rizwan, M. Farid and M.K. Irshad (2016). Phosphorus amendment decreased cadmium (Cd) uptake and ameliorates chlorophyll contents, gas exchange attributes, antioxidants, and mineral nutrients in wheat (Triticum aestivum L.) under Cd stress. Arch. Agron. Soil Sci. 62:533-546.

Aslam, M., Q. Sohail, M. Maqbool, S. Ahmad and R. Shahzad (2017). Combining ability analysis for yield traits in diallel crosses of maize. J. Anim. Plant Sci. 27:136-143.

Björn, L.O., G.C. Papageorgiou and R.E. Blankenship. 2009. A viewpoint: why chlorophyll a? Photosynth. Res. 99:85-98.

Brauman, K.A., S. Siebert and J.A. Foley (2013). Improvements in crop water productivity increase water sustainability and food securitya global analysis. Environ. Res. Lett. 8:024-030.

Cai, C., G. Zhu, T. Zhang and W. Guo. 2017. Highdensity $80 \mathrm{~K} \mathrm{SNP}$ array is a powerful tool for genotyping $G$. hirsutum accessions and genome analysis. BMC Genomics. 18:654.

Chourasia, K.N. (2017). Resistance/Tolerance mechanism under water deficit (Drought) condition in plants. Int. J. Curr. Microbiol. App. Sci. 6:66-78.

Costache, M.A., G. Campeanu and G. Neata (2012). Studies concerning the extraction of chlorophyll and total carotenoids from vegetables. Rom. Biotech. Lett. 17:7703-7708.

Davis, K.F., M.C. Rulli, F. Garrassino, D. Chiarelli, A. Seveso and P. D'odorico (2017). Water limits to closing yield gaps. Adv. Water Res. 99:67-75.

Edreva, A. (2005a). Generation and scavenging of reactive oxygen species in chloroplasts: a submolecular approach. Agr. Ecosys. Environ. 106:119-133.

Edreva, A. (2005b). The importance of nonphotosynthetic pigments and cinnamic acid derivatives in photoprotection. Agr. Ecosys. Environ. 106:135-146.

El-Tayeb, M. (2006). Differential responses of pigments, lipid peroxidation, organic solutes, catalase and per-oxidase activity in the leaves of two Vicia faba L. cultivars to drought. Int. J. Agric. Biol. 8:116-122.

Fang, Y. and L. Xiong (2015). General mechanisms of drought response and their application in drought resistance improvement in plants. Cell. Mol. Life Sci. 72:673-689.

Ghahfarokhi, G., M., S. Mansurifar, R. TaghizadehMehrjardi, M. Saeidi, A.M. Jamshidi and E. Ghasemi (2015). Effects of drought stress and rewatering on antioxidant systems and relative water content in different growth stages of 
maize (Zea mays L.) hybrids. Arch. Agron. Soil Sci. 61:493-506.

Government of Pakistan. 2016-17. Pakistan Economic Survey. Ministry of Finance, Economic Advisor's Wing, Islamabad.

Han, J.-M., H.-F. Meng, S.-Y. Wang, C.-D. Jiang, F. Liu, W.-F. Zhang and Y.-L. Zhang (2016). Variability of mesophyll conductance and its relationship with water use efficiency in cotton leaves under drought pretreatment. J. Plant Physio. 194:61-71.

He, J., D. Gu, X. Wu, K. Reynolds, X. Duan, C. Yao, J. Wang, C.-S. Chen, J. Chen and R.P. Wildman (2005). Major causes of death among men and women in China. N. Engl. J. Med. 353:11241134.

Jaleel, C.A., P. Manivannan, A. Wahid, M. Farooq, H.J. Al-Juburi, R. Somasundaram, and R. Panneerselvam (2009). Drought stress in plants: a review on morphological characteristics and pigments composition. Int. J. Agric. Biol. 11:100-105.

Javaid, A., F.M. Azhar, I.A. Khan and S.A. Rana (2014). Genetic basis of some yield components in Gossypium hirsutum L. Pakistan J. Agri. Sci. 51:143-146.

Kalaji, H.M., A. Jajoo, A. Oukarroum, M. Brestic, M. Zivcak, I.A. Samborska, M.D. Cetner, I. Łukasik, V. Goltsev and R.J. Ladle (2016). Chlorophyll a fluorescence as a tool to monitor physiological status of plants under abiotic stress conditions. Acta Physiol. Plant. 38:102. https://doi.org/10.1007/s11738-016-2113-y

Karimi, S., A. Yadollahi, K. Arzani, A. Imani and M. Aghaalikhani (2015). Gas-exchange response of almond genotypes to water stress. Photosynthetica. 53:29-34.

Kempthorne, O. 1957. An Introduction to Genetic Statistics. New York, John Wiley and Sons, Inc., New York.

Khan, A., D.K.Y. Tan, M.Z. Afridi, H. Luo, S.A. Tung, M. Ajab and S. Fahad (2017). Nitrogen fertility and abiotic stresses management in cotton crop: a review. Environ. Sci. Poll. Res. 24:1455114566.

Kirda, C., S. Topcu, H. Kaman, A. Ulger, A. Yazici, M. Cetin and M. Derici (2005). Grain yield response and $\mathrm{N}$-fertiliser recovery of maize under deficit irrigation. Field Crops Res. 93:132141.

Leopoldini, M., N. Russo, S. Chiodo and M. Toscano (2006). Iron chelation by the powerful antioxidant flavonoid quercetin. J. Agric. Food Chem. 54:6343-6351.

Li, Y., H. Li, Y. Li and S. Zhang (2017). Improving water-use efficiency by decreasing stomatal conductance and transpiration rate to maintain higher ear photosynthetic rate in droughtresistant wheat. Crop J. 5:231-239.

Luo, H., Y. Zhang and W. Zhang (2016). Effects of water stress and rewatering on photosynthesis, root activity, and yield of cotton with drip irrigation under mulch. Photosynthetica. 54:65-73.

Mahawar, S., I. Katageri and M. Jadhav. 2017. Evaluation and Characterization of Genetically Modified Cotton Gossypium herbaceum var. Jayadhar for Helicoverpa armigera Resistance. Int. J. Curr. Microbiol. App. Sci. 6:2780-2792.

Maqsood, R.H., I. Khaliq, M. Kashif and N. Ahmed. 2018. Gene action array related to morphophysiological traits using population genetics in Triticum aestivum L. Pakistan J. Agric. Sci. 55:513-519.

Miner, G.L., W.L. Bauerle and D.D. Baldocchi (2017). Estimating the sensitivity of stomatal conductance to photosynthesis: a review. Plant Cell Env. 40:1214-1238.

Munir, S., M.K. Qureshi, A.N. Shahzad, H. Manzoor, M.A. Shahzad, K. Aslam and H.-U.-R. Athar (2018). Assessment of gene action and combining ability for fibre and yield contributing traits in interspecific and intraspecific hybrids of cotton. Czech J. Genet. Plant Breed. 54:71-77.

Osakabe, Y., K. Osakabe, K. Shinozaki and L.S. Tran (2014). Response of plants to water stress. Front. Plant Sci. 5. doi: 10.3389/fpls.2014.00086.

Osório, M.L., J. Osório, A.C. Vieira, S. Gonçalves and A. Romano. (2011). Influence of enhanced temperature on photosynthesis, photooxidative damage, and antioxidant strategies in Ceratonia siliqua L. seedlings subjected to water deficit and rewatering. Photosynthetica. 49:3-12.

Ozdemir, E.O. and B. Sade. 2019. Linex Tester Analysis of Stomatal Conductance, Chlorophyll Content, Photosynthetic Efficiency, and Transpiration Rate Traits in Maize. Maydica. 64:8

Parveen, N., A. Shakeel, T.A. Malik and F.S. Awan. 2019. Genetic studies for improving seed yield and quality traits including carotenoids, chlorophyll and protein. Pakistan J. Bot. 51:2105-2110.

Prochazkova, D., R. Sairam, R. G. Srivastava, and D. Singh (2001). Oxidative stress and antioxidant activity as the basis of senescence in maize leaves. Plant Sci. 161:765-771.

Rehman, A., A. Shakeel, M. T. Azhar and I. Haq (2017). Biplot and cluster analysis of some water stress tolerance related traits in upland cotton. J. Environ. Agric., 2: 146-156.

Sarwarkar, M., A. Solanke, G.S. Mhasal and S.B. Deshmukh. (2015). Combining ability and 
heterosis for seed cotton yield, its components and quality traits in (Gossypium hirsutum L.). Indian. J. Agric. Res., 49: 154-159.

Shah, A.R., T.M. Khan, H.A. Sadaqat and A.A. Chatha. 2011. Alterations in leaf pigments in cotton (Gossypium hirsutum) genotypes subjected to drought stress conditions. Int. J. Agric. Biol. 13:902-908

Shakoor, M.S., T.A. Malik, F.M. Azhar and M.F. Saleem (2010). Genetics of agronomic and fiber traits in upland cotton under drought stress. Int. J. Agric. Biol. 12:495-500.

Steel, R.G. and J.H. Dickey (1997). Principles and procedures of statistics: A biometrical approach. (3rd ed.) McGraw Hill, New York.

Ullah, I. and Y. Zafar (2006). Genotypic variation for drought tolerance in cotton (Gossypium hirsutum L.): seed cotton yield responses. Pakistan J. Bot. 38: 1679-1687.

Wahid, A. and A. Ghazanfar (2006). Possible involvement of some secondary metabolites in salt tolerance of sugarcane. J. Plant Physiology. 163:723-730.

Xiong, Q., B. Wang, and C. Duan (2000). Molecular mechanism of water-stress response in plant. Prog. Bioch. Biophy. 27:247-250.

Yu, L.H., S.J. Wu, Y.S. Peng, R.N. Liu, X. Chen, P. Zhao, P. Xu, J.B. Zhu, G.L. Jiao and Y. Pei (2016). Arabidopsis EDT1/HDG11 improves drought and salt tolerance in cotton and poplar and increases cotton yield in the field. Plant Biotechnol. J. 14:72-84. 